\title{
Circuit
}

Musiques contemporaines

\section{Repères biographiques et catalogue des oeuvres}

Volume 5, numéro 1, 1994

Gilles Tremblay : réflexions

URI : https://id.erudit.org/iderudit/902096ar

DOI : https://doi.org/10.7202/902096ar

Aller au sommaire du numéro

Éditeur(s)

Les Presses de l'Université de Montréal

ISSN

1183-1693 (imprimé)

1488-9692 (numérique)

Découvrir la revue

Citer ce document

(1994). Repères biographiques et catalogue des oeuvres. Circuit, 5(1), 69-72.

https://doi.org/10.7202/902096ar

Ce document est protégé par la loi sur le droit d'auteur. L'utilisation des services d'Érudit (y compris la reproduction) est assujettie à sa politique d'utilisation que vous pouvez consulter en ligne.

https://apropos.erudit.org/fr/usagers/politique-dutilisation/ 


\section{GILLES TREMBLAY: DOCUMENTATION} REPÈRES BIOGRAPHIQUES ET CATALOGUE DES OEUVRES

1932

Naissance le 6 septembre à Arvida. Son père, Thomas-Léon Tremblay, est industriel ef entomologiste amateur et sa mère Gilberte écrit des livres pour enfants. Gilles est l'aîné d'une famille de cinq enfants: René, industriel de la région de Chicoutimi, Alain, céramiste, Dominique, violoniste et Michel, graveur.

1944...

Études au collège Stanislas et au collège Brébeuf. II terminera ses études classiques en cours privés.

Cours de peinture avec le frère Jérôme, peintre pédagogue et ami de Borduas. «e voulais que les couleurs bougent. C'est le goût du mouvement qui m'a amené à la musique ». (Émission Rencontres, 1974).

Début d'une longue amitié avec l'astrophysicien Hubert Reeves avec lequel il partage son amour de la nature.

\section{4-1949}

Études privées en musique avec Jocelyne Binet (piano), Edmond Trudel (piano), Gabriel Cusson (Culture de l'oreille).

\section{$1949-1954$}

Études au Conservatoire de musique de Montréal avec Isabelle Delorme (solfège),
Jean Papineau-Couture (acoustique), Edmond Trudel puis Germaine Malépart (piano).

Cours privés de composition et d'écriture avec Claude Champagne, dont les fonctions d'administrateur lui interdisaient d'assumer des cours réguliers au Conservatoire.

\section{0}

Cours d'été au Malboro School of Music (Vermont) alors sous la direction de Rudolf Serkin, Marcel Moïse et Adolf Buch. Il étudie avec Louis Moïse (fils de Marcel), pianiste et flûtiste.

1951

Cours d'été au Malboro School of Music (Vermont).

\section{2}

Rencontre Varèse à New York. «ll représente beaucoup pour moi. Je l'ai connu d'abord par le disque, grâce à François Morel, alors que j'étais étudiant. Ce fut une révélation. J'ai trouvé là un langage d'une poésie extraordinaire et d'une grande efficacité.» Entrevue avec Yvon Paré, Le Quotidien, 14 septembre 1979). «C'est par un ami de la famille, Thomas Greenwood, professeur de littérature anglaise à l'Université de Montréal, que je fus introduit à Varèse à New York.» (Entrevue avec
Maryvonne Kendergi, Anthologie RadioCanada International, 1983).

\section{$1952-1953$}

Cours d'histoire de la musique à l'Université de Montréal avec Jean Vallerand.

\section{3}

Cours d'été au Malboro School of Music au Vermont.

Premier prix de piano au Conservatoire de musique du Québec.

\section{3-1954}

Cours de contrepoint avec Jocelyne Binet.

\section{4}

II participe, le $1^{\text {or }}$ mai, avec Serge Garant ef François Morel, au premier concert de musique contemporaine, au Conservatoire de musique de Montréal.

En septembre, départ pour Paris sur le paquebot a liberté * à bord duquel il rencontre à nouveau Varèse qui lui montre la partition de Déserts qui sera créée à Paris quelques semaines plus tard...

\section{4-1957}

II s'inscrit au Conservatoire National Supérieur de Musique de Paris. Études de piano 
et d'écriture avec Yvonne Loriod, de contrepoint avec Andrée Vaurabourg-Honegger, des ondes Martenot avec Maurice Martenot (1956-1958) et d'analyse avec Olivier Messiaen. Il découvre la musique de Pierre Boulez dont il avait connu les premières œuvres par Serge Garant puis celles de Stockhausen et de Xenakis.

Récitals de piano.

\section{7}

Premier prix d'analyse du Conservatoire national supérieur de Musique de Paris.

Cours d'été à Darmstadt avec Stockhausen, aux Ferienkurse für Neue Musik grâce à une bourse du Kranichsteiner Musik Institut.

Le 24 août, il épouse Jacqueline Pinel, peintre ef fondatrice en 1965 de l'École Buissonnière, consacrée à l'éveil des enfants par les arts.

\section{8}

En mai, il obtient une première médaille d'ondes Martenot au Conservatoire National Supérieur de Musique de Paris.

En juin, il obtient une licence en contrepoint de l'École normale supérieure de Musique de Paris où il étudie (1957-1958) avec Madame Andrée Vaurabourg-Honegger. Naissance de sa fille Joëlle.

\section{8-1961}

Boursier du Conseil des Arts du Canada.

\section{9}

Création en mai de Phases et Réseaux à Cologne par Yvonne Loriod.

Stage da l'ORTF à Paris au GRM sous la direction de Pierre Schaeffer.

Donne des cours d'analyse au Centre d'arts d'Orford, JMC, durant l'été.
Cours d'été à Darmstadt avec Pierre Boulez et Henri Pousseur, grâce d̀ une bourse du Kranichsteiner Musik Institut.

Le 17 octobre, naissance à Paris de son fils Jean-François.

\section{1}

Retour définitif à Montréal. II devient membre de la ligue canadienne de compositeurs.

Donne des cours d'analyse au Centre d'arts d'Orford, JMC, durant l'été et présente un concert de musique concrète et électronique lœuvres de Berio, Boucourechliev, Chamass, Ferrari, Schaeffer, Stockhausen et Xenakis).

\section{$1961-1966$}

Donne des cours d'analyse au Conservatoire de musique de Québec.

\section{1}

Il collabore avec le poète Fernand Ouellette à une série de treize émissions radiophoniques intitulée Paroles de poètes à RadioCanada, pour laquelle il compose la musique.

\section{2}

Création le 26 juillet de Mobile au Centre d'Art Orford par Calvin Sieb et Irving Heller.

\section{2...}

Nommé professeur d'analyse au Conservatoire de musique de Montréal.

\section{3}

Création le 24 mars de Cantique de durées au Domaine musical (Paris) sous la direction d'Ernest Bour.
Participe comme ondiste à la première canadienne de la Turangalîla-Symphonie de Messiaen avec l'Orchestre symphonique de Toronto.

\section{5}

Création en février de Champs I à Montréal par l'Ensemble de percussions de Paris.

\section{6}

Naissance de son fils Emmanuel.

Création le 25 février de Kekoba à Montréal, R.-C. sous la direction de Gilles Tremblay.

$1967 .$.

Enseigne la composition en plus de l'analyse au Conservatoire de musique de Montréal. Création de Centre-Élan au Pavillon du Québec, durant l'Exposition universelle.

Création le 21 mars de Souffles (Champs II) à Montréal par la SMCQ sous la direction de Serge Garant.

\section{8}

Prix Calixa-Lavallée de la Société Saint-JeanBaptiste, pour la sonorisation du Pavillon du Québec, durant l'Exposition universelle de 1967.

Membre du conseil d'administration de la SMCQ.

Il prend part au colloque sur le Canada à Cerisy-la-Salle, France (22-31 juillet) où il fait la connaissance du poète Eugène Guillevic et présente les œuvres de quelques compositeurs québécois. 
1969

Création le 2 août de Vers (Champs III) à Strafford sous la direction de lawrence Smith.

\section{0}

Création le 22 octobre de Dimension Soleils concert de la SMCQ.

\section{1}

Dirige Kekoba à New York dans le cadre du New Image of Sound, at Hunter College. Le 27 août, naissance de son fils Guillaume. Membre du jury du Concours international de composition à la biennale de Paris.

Création le $1^{\text {er }}$ mars de ... le siffllement des vents porteurs de l'amour... au Centre national des Arts d'Ottawa par Robert Cram et lan Bernard.

\section{2}

Membre du jury du Concours international de flûte au $9^{\circ}$ Festival d'art contemporain de Royan.

De juin à septembre, voyage d'études en Extrême-Orient (Japon, Corée, Philippines, Chine, Java, Bali, Inde) grâce à une bourse du Conseil des Arts.

Création le 17 mai de Solstices à Montréal sous la direction de Gilles Tremblay.

\section{2-1981}

Membre du Conseil de Pastorale du diocèse de Montréal.

\section{3}

Reçoit la médaille du Conseil canadien de la musique.
Création le 23 avril de Jeux de Solstices au Centre national des Arts d'Ottawa sous la direction de Mario Bernardi.

\section{5}

Membre du jury de la Société internationale de musique contemporaine, présidée par lannis Xenakis.

Création le 8 février de Oralléluiants à Toronto par le New Music Concerts sous la direction de Gilles Tremblay.

\section{5-1976}

Membre de la Commission consulfative du Conseil des Arts du Canada.

\section{6}

Création en juillet 1976 de Traçantes, auprès, au loin... d̀ La Rochelle au Concours international de piano contemporain.

Membre du jury au Concours international de piano contemporain.

Controverse avec le chef d'orchestre Raphaël de Burgos au sujet de la création de Fleuves.

\section{7}

Création le 3 mai de Fleuves par l'Orchestre symphonique de Montréal sous la direction de Serge Garant.

\section{8}

Nommé compositeur de l'année par le Conseil canadien de la musique.

Création le 11 mars de Vers le soleil à Paris par le Nouvel Orchestre Philharmonique de Radio-France sous la direction de Théodore Antoniou.
Création le 30 novembre de Compostelle 1 par l'ensemble $2 e 2 m$ du Collectif musical international de Champigny sous la direction de Paul Méfano.

\section{9}

Président du Conseil d'administration de «Traditions musicales du monde *, avec José Evangelista, Ramon Pelinski ef John Rea.

\section{0}

Séjour en Israël et en Égypte.

Professeur invité à l'University of British Columbia.

\section{1}

Création le 12 avril de DZEl au Vancouver New Music Society par l'Ensemble Gropus 7. Création le 8 octobre du Signe du lion à Montréal par la SMCQ sous la direction de Serge Garant.

\section{2}

En juin, au lendemain du rapatriement unilatéral de la Constitution, il refuse l'Ordre du Canada pour des raisons politiques et symboliques.

Président du jury pour le Concours des jeunes compositeurs de Radio-Canada.

\section{2-1988}

Président du Conseil d'administration de la SMCQ. 
1983

Édition de l'Anthologie de la musique canadienne, consacrée au compositeur par RadioCanada International.

Compositeur invité à I'University of Harfford, Connecticut.

Création le 17 février de Envoi à Montréal par la SMCQ sous la direction de Serge Garant et le pianiste Claude Helffer.

\section{4}

Création au printemps de Envol par JeanFrançois Tremblay.

\section{5}

Voyage au Pérou en août et découverte de ses civilisations anciennes.

Création le 13 avril de Triojubilus à Toronto par le New Music Concerts.

\section{6}

Création en juillet des Vêpres de la Vierge d̀ l'Abbaye de Sylvanès (France) par le chœur de l'orchestre de Lyon /direction Bernard Tétu), l'Ensemble des jeunes instrumentistes du Québec, Pauline Vaillancourt, Réjean Poirier et Jean-François Tremblay sous la direction de Gilles Tremblay.
Création le 9 avril de Un 9 par les musiciens des événements du Neuf.

\section{$1987-1988$}

Directeur artistique de la SMCQ.

1988

Adhère à l'Association des artistes pour la paix.

Création le 18 octobre de Katadrone (Contrecri) à Montréal par le Scottish Symphony Orchestra sous la direction de Jerzy Maksymiuk.

\section{9}

Invité à la Banff School of the Arts.

Nommé membre correspondant de la Société française d'analyse musicale. Participe au premier congrès européen d'analyse musicale à Colmar.

Participe aux rencontres du Centre JacquesCartier de Lyon.

Création le 5 juin de Cèdres en voiles Threne pour le Liban à Montréal par Emmanuel Tremblay.

\section{0}

Président du jury du concours radiophonique des jeunes compositeurs, organisé par Radio-Canada.

\section{1}

Devient membre de l'Ordre du Québec. Reçoit le Prix du Québec « Denise-Pelletier *. Création le 3 mars de Aubes ou Initial à Ottawa par l'Ensemble Pierrôt.

Création le 2 juin de Musique du feu à Montréal par la SMCQ sous la direction de Walter Boudreau.

\section{2}

Nomination par Jack Lang, ministre de l'Éducation nationale et de la Culture (République française) au grade de Chevalier de l'Ordre des Arts et des Lettres.

Création le 12 octobre de AVEC, wampum symphonique à Montréal par l'Orchestre Symphonique de Montréal sous la direction de Charles Dutoit.

\section{3}

Nommé « Artiste de la Paix 1992 » pour son œuvre AVEC, wampum symphonique. Création le 5 mai de Vers une étoile pour orgue à Montréal par Bernard Focroulle. 